\title{
Multi-wavelength time-resolved NIRS measurements for estimation of absolute concentration of chromophores: blood phantom study
}

\begin{abstract}
Aleh Sudakou, Frederic Lange, Helene Isler, Anna Gerega, Daniel Ostojic, et al.
\end{abstract}

Aleh Sudakou, Frederic Lange, Helene Isler, Anna Gerega, Daniel Ostojic, Piotr Sawosz, llias Tachtsidis, Martin Wolf, Adam Liebert, "Multi-wavelength time-resolved NIRS measurements for estimation of absolute concentration of chromophores: blood phantom study," Proc. SPIE 11074, Diffuse Optical Spectroscopy and Imaging VII, 1107422 (11 July 2019); doi: $10.1117 / 12.2527215$ 


\title{
Multi-wavelength time-resolved NIRS measurements for estimation of absolute concentration of chromophores: blood phantom study
}

\author{
Aleh Sudakou ${ }^{a^{*}}$, Frederic Lange ${ }^{\mathrm{b}}$, Helene Isler ${ }^{\mathrm{c}}$, Anna Gerega ${ }^{\mathrm{a}}$, Daniel Ostojic ${ }^{\mathrm{c}}$, \\ Piotr Sawosz ${ }^{\mathrm{a}}$, Ilias Tachtsidis ${ }^{\mathrm{b}}$, Martin Wolf ${ }^{\mathrm{c}}$, Adam Liebert ${ }^{\mathrm{a}}$. \\ a) Laboratory for Biomedical Optics, Nalecz Institute of Biocybernetics and Biomedical Engineering Polish \\ Academy of Sciences (IBIB PAN), Poland. \\ b) Biomedical Optics Research Laboratory, Department of Medical Physics and Biomedical Engineering, \\ University College London (UCL), United Kingdom. \\ c) Biomedical Optics Research Laboratory, University Hospital Zürich (USZ), University of Zurich, Switzerland.
}

\begin{abstract}
Absolute concentrations of oxyhaemoglobin and deoxyhaemoglobin obtained using multi-wavelength measurements of time-resolved diffuse reflectance signals are presented. The aim was to test how accurately the concentration of oxy- and deoxyhemoglobin can be measured simultaneously at multiple wavelengths. The optical signals were collected using the system constructed by the author's group ${ }^{\text {a }}$, which records the distribution in time of flight of photons (DTOFs) simultaneously at 16 spectral channels ranging from 650 to $850 \mathrm{~nm}$. The measurements were carried out on liquid phantoms containing intralipid solution, human blood and yeast in varying amounts. The oxygen saturation of blood covered a continuous range from $100 \%$ to $0 \%$ during 6 deoxygenation cycles. The estimated values of total haemoglobin $(20.9,35.7,57.3,45.7 \mu \mathrm{M})$ are close to the reference values obtained using a blood gas analyzer $(21.3,37.0,57.3,48.0 \mu \mathrm{M})$ and the estimated values of absolute concentrations of oxy-, deoxyand total haemoglobin are similar to the values obtained using a commercial frequency-domain NIRS system $\left(\right.$ OxiplexTS ${ }^{\mathrm{TM}}$ ). The phantom measurements have shown the capability to measure the absolute concentrations of chromophores in a studied media using multi-wavelength, time-resolved NIRS technique. The excess number of spectral channels can potentially be used to resolve changes in oxidation state of cytochrome-c-oxidase enzyme.
\end{abstract}

Keywords: diffuse optics, time-resolved NIRS, blood phantom, oxygen saturation.

\section{INTRODUCTION}

Time-resolved near-infrared spectroscopy (TR-NIRS) can provide information about absolute concentrations of oxyhaemoglobin ( $\mathrm{CHьO2)}$ and deoxyhaemoglobin $(\mathrm{CHb})$ in the studied tissue and hence help assess the tissue blood oxygen saturation $\left(\mathrm{StO}_{2}=\mathrm{CHbO2} / \mathrm{CHbT}\right.$, where $\left.\mathrm{CHbT}_{\mathrm{H}}=\mathrm{CHbO2}_{\mathrm{H}}+\mathrm{CHb}\right)$ with high temporal resolution $(3 \mathrm{~Hz})$, non-invasively, continuously for many hours, with non-ionizing radiation and at the bedside [1]. The in-vitro measurements on blood-lipid phantoms were employed to compare continuous wave to frequency-domain commercial oximeter devices, showing the differences between the values obtained by these devices subjected to the same measurement conditions [2]. The blood-lipid phantoms and the well-established protocols for dynamic deoxygenation of blood can be used to test a methodology for estimation of oxygen saturation [2].

We present the estimation of $\mathrm{CHьO2,} \mathrm{Снь,} \mathrm{Сньт} \mathrm{and} \mathrm{hence} \mathrm{StO}_{2}$ using our multi-wavelength time-resolved NIRS system [3] in a series of dynamic blood-lipid phantoms carried out at $\mathrm{USZ}^{\mathrm{C}}$ and partially repeated at IBIB ${ }^{\mathrm{a}}$. The phantom measurements were partially repeated also at $\mathrm{UCL}^{\mathrm{b}}$ with their multi-wavelength time-resolved NIRS system [4]. The minimum of 2 wavelengths is required for the calculation of concentration of two chromophores. The excessive number of spectral channels potentially could be used for assessment of depth-resolved changes in oxidation state of cytochrome-c-oxidase enzyme $\left(\Delta \mathrm{C}_{\mathrm{CCO}}\right)$ during changes in the cerebral oxygen delivery.

Diffuse Optical Spectroscopy and Imaging VII, edited by Hamid Dehghani, Heidrun Wabnitz, Proc. of SPIE-OSA Vol. 11074, 1107422 · C 2019 SPIE-OSA · CCC code: 1605-7422/19/\$21 · doi: 10.1117/12.2527215 


\section{METHOD}

\subsection{Experimental:}

The phantom consisted of a 3D printed container with $1 \mathrm{~mm}$ thick silicone windows $\left(\mu \mathrm{a}=0.63 \mathrm{~mm}^{-1}, \mu \mathrm{s}^{\prime}=6 \mathrm{~mm}^{-1}\right.$ at $690 \mathrm{~nm}$ mimicking optical properties of fat) filled with homogeneous liquid containing saline $(2.5 \mathrm{~L})$, Fresenius Kabi Intralipid $200 \mathrm{mg} / \mathrm{ml}$, blood and yeast $(3.0 \mathrm{~g})$. In the first phantom measurement, $74 \mathrm{ml}$ of intralipid was used and blood was added before the start of each oxygenation cycle resulting in 20,35 and $55 \mathrm{ml}$ of blood. In the second phantom measurement, $45 \mathrm{ml}$ of blood was used and intralipid was added before the start of each oxygenation cycle resulting in 75,100 and $125 \mathrm{ml}$ of intralipid. The optical properties resulting from $74 \mathrm{ml}$ of intralipid and $45 \mathrm{ml}$ of blood are close to the typical optical properties of tissue. The blood was deoxygenated by adding yeast and was again oxygenated by bubbling oxygen from an oxygen bottle. The oxygen saturation covered a continuous range from $100 \%$ to $0 \%$. The following were controlled: $7.4 \mathrm{pH}$ level using sodium bicarbonate buffer, $37^{\circ} \mathrm{C}$ temperature and a constant mixing speed of $500 \mathrm{rpm}$ using a hotplate with a magnetic stirrer.

\subsection{Data analysis: methodology of TR-NIRS for estimation of absolute concentrations}

Measurements were performed with the multi-wavelength TR-NIRS system that was developed by the authors group ${ }^{\mathrm{a}}$. The system records the distribution of times of flight (DTOFs) of photons simultaneously at 16 spectral channels of approximately $12.5 \mathrm{~nm}$ width from 650 to $850 \mathrm{~nm}$. The source and detector fibers were positioned $3 \mathrm{~cm}$ apart and the acquisition time was 0.3 seconds. The silicone window in the phantom measurements had low optical density and thickness, hence for simplification the medium was assumed homogeneous. The absolute absorption and the reduced scattering coefficients were calculated based on statistical moments of DTOFs [3]: the mean time of flight of photons and the variance of the DTOF corrected for the influence of the instrument response function (IRF). The calculated absorption coefficients of each spectral channel were adjusted for the absorption of water. Then the Beer-Lambert law was applied to retrieve absolute concentrations of oxy- and deoxyhaemoglobin. The final results were smoothed by applying a moving average with time window of 4.8 seconds (16 data points). The commercially available OxiplexTS ${ }^{\mathrm{TM}}$ (frequency-domain, 692 and $834 \mathrm{~nm}$, ISS, Champaign, Illinois, USA) was used to record the data simultaneously to obtain reference values for oxy-, deoxy- and total haemoglobin. A blood gas analyzer (ABL90 FLEX, Radiometer Medical ApS, Brønshøj, Denmark) was employed on human blood samples from the erythrocyte bag to calculate the concentration of total haemoglobin in the blood. Subsequently, Сньт was calculated based on the concentration of total haemoglobin and the strength of dilution in the liquid phantom.

\section{RESULTS AND CONCLUSION}

The results of the multiwavelength TR-NIRS system are shown in figure 1. For different concentrations of blood (fig. 1), the mean values of Сньт $(22.1 \pm 1.3,37.4 \pm 1.4$ and $58.3 \pm 2.4 \mu \mathrm{M})$ during three deoxygenation cycles correspond well to the values obtained by co-oximetry $(21.3 \pm 0.1,37.0 \pm 0.2$ and $57.3 \pm 0.3 \mu \mathrm{M})$. For different concentrations of intralipid (results not shown), the average values of Сньт $(46.2 \pm 1.2,44.3 \pm 1.4$ and $46.5 \pm 1.9 \mu \mathrm{M})$ are close to the value obtained by co-oximetry $(48.0 \pm 0.3 \mu \mathrm{M})$. The estimation of absolute concentration of haemoglobin seems to have negligible dependence on the background reduced scattering coefficient for scattering values close to those of typical tissue.

For low concentration of haemoglobin (reflected in low absorption) the values of oxygen saturation measured by TR-NIRS system ${ }^{a}$ range only from $82 \%$ to $18 \%$. However, when the concentration of blood is increased and closer resembles average tissue, the range of values of oxygen saturation increases and ranges from $100 \%$ to $0 \%$. The amount of intralipid (reflected in reduced scattering coefficient) has no apparent effect on the estimation of absolute concentrations and oxygen saturation. 


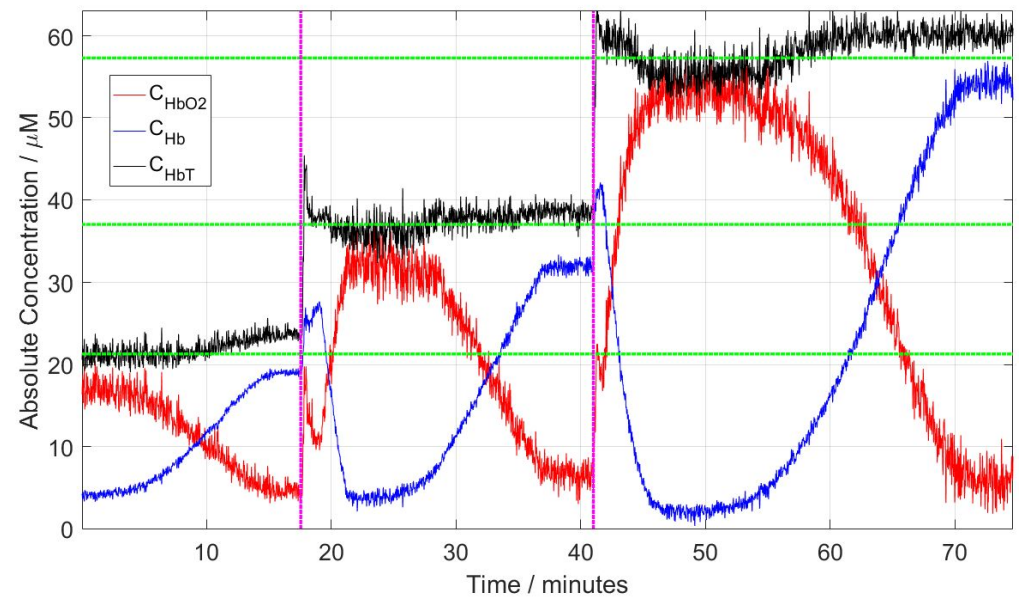

Figure 1. Absolute concentrations of $\mathrm{CHbO2}, \mathrm{CHb}$ and $\mathrm{CHbT}$ in a phantom with varying amount of blood (20, 35, 55 ml). The vertical magenta lines correspond to times when blood or intralipid was added followed by oxygenation of blood. The values of $\mathrm{CHbT}$ calculated with the result of a blood gas analyzer are: $21.3,37.0$ and $57.3 \mu \mathrm{M}$ (horizontal green lines) for the three deoxygenation cycles.

In conclusion, the phantom measurements have shown the performance of our TR-NIRS system for various background optical properties and for a continuous range of oxygen saturation values.

Further studies will aim to estimate changes in oxidation state of cytochrome-c-oxidase enzyme by exploiting the excess number of spectral channels in our system. Also, the methodology will be improved to provide depth-resolved measurements in order to separate signals originating from different depths, i.e. in the brain and in the extracerebral tissues.

\section{ACKNOWLEDGMENTS}

This project has received funding from the European Union's Horizon 2020 Marie Skłodowska-Curie Innovative Training Networks (ITN-ETN) programme, under grant agreement no 675332, BitMap.

\section{REFERENCES}

[1] Bale G., Elwell CE., Tachtsidis I., "From Jobsis to the present day: a review of clinical near-infrared spectroscopy measurements of cerebral cytochrome-c-oxidase," J Biomed Opt, 21(9): p. 091307, (2016).

[2] Kleiser, S., Nasseri N., Andresen B., Greisen B., Wolf M., "Comparison of tissue oximeters on a liquid phantom with adjustable optical properties," Biomed Opt Express, 7(8): p. 2973-92, (2016).

[3] Gerega, A., Milej D., Weigl W., Kacprzak M., Liebert A., "Multiwavelength time-resolved near-infrared spectroscopy of the adult head: assessment of intracerebral and extracerebral absorption changes," Biomed. Opt. Express, 9(7): p. 2974--2993, (2018).

[4] Lange, F., Dunne L., Hale L., Tachtsidis I., "MAESTROS: A Multiwavelength Time-Domain NIRS System to Monitor Changes in Oxygenation and Oxidation State of Cytochrome-C-Oxidase," IEEE Journal of Selected Topics in Quantum Electronics, 25(1): p. 1-12, (2019). 